\title{
De Kyoto à Buenos Aires : l'émergence d'un nouveau contexte pour la compétition industrielle
}

\author{
Jean-Charles Hourcade \\ Directeur de Recherche Cnrs, directeur du Cired (Ehess, URA 940 Cnrs) \\ Frédéric Ghersi \\ Stagiaire de Recherche au Cired
}

\section{Introduction : un bref retour historique de Rio de Janeiro à Kyoto}

Parce qu'il ne faisait qu'évoquer l'objectif, pour les pays dits de l'Annexe 1 (OCDE et pays en transition), de la stabilisation en 2000 de leurs émissions de gaz à effet de serre (GES) au niveau atteint en 1990, le résultat de la conférence de Rio de Janeiro en juin 1992 n'a peutêtre pas été perçu avec le sérieux qu'il méritait. Cinq ans de débats allaient s'ensuivre à l'OCDE, au Groupe Intergouvernemental d'Experts pour l'étude des Climats (GIEC) et sur diverses scènes scientifiques, quant aux formes à donner à la coordination internationale des efforts et au degré d'ambition de l'action. Ce processus vient de déboucher à Kyoto en décembre 1997 sur un protocole qui risque de changer la donne de la compétition industrielle internationale. On ne peut en comprendre les implications sans revenir brièvement sur sa genèse ${ }^{1}$.

Dès 1990, une vive confrontation a opposé deux approches à la négociation : devait-on rechercher un accord autour de politiques et mesures harmonisées sans fixer d'objectifs quantifiés, ou un engagement autour de tels objectifs en laissant aux Etats le soin de mener les politiques qu'ils jugeraient les plus adaptées ? La première approche, officiellement soutenue par la France et les autres pays européens, était justifiée par la difficulté de prédire les résultats des politiques et mesures et par l'impossibilité de trouver des règles de répartition des objectifs qui soient reconnues comme équitables ${ }^{2}$. C'est pourtant la deuxième qui allait l'emporter lors de l'adoption du Mandat de Berlin en 1994. Il était vite devenu évident en effet qu'en dehors de mesures sur les soutes maritimes et aériennes, la seule mesure globale susceptible de coordonner des actions décentralisées était une taxe internationale sur les émissions de carbone. Les administrations Bush et Clinton ont tour à tour jugé politiquement inacceptable une telle perspective, en raison du réflexe anti-taxe prévalant aux USA, et de l'atteinte à la souveraineté nationale qu'aurait représentée une taxe coordonnée, argument partagé par les Britanniques. L'idée de permis d'émission négociables (PEN) internationaux offrait ici une garantie de constitutionnalité et surtout la possibilité d'un compromis acceptable par les milieux industriels les plus réticents à toute action significative en permettant une «where flexibility». Or l'instauration de PEN supposait évidemment une allocation initiale des droits, donc une négociation sur des objectifs quantifiés.

In fine, le retrait de la proposition européenne de taxe mixte carbone-énergie en 1992, pour des raisons qu'il n'est pas lieu de développer ici, ouvrit définitivement la voie à une approche par des Quantified Emission Limitation and Reduction Objectives (Qelros) contraignants, qui allaient vider de sens, sans que toutes les parties prenantes en soient conscientes en Europe, toute négociation sur des politiques coordonnées (on ne peut planifier

\footnotetext{
${ }^{1}$ On en trouvera un historique plus développé in Godard (1997) et Hourcade (1997)

${ }^{2}$ Un bilan des débats économiques autour de ces deux approches peut être lu in OCDE (1993)
} 
à la fois en quantités et en prix), et placer les permis d'émission négociables au cœur des débats à la Conférence de Kyoto. Après avoir résumé les résultats de cette négociation, nous soulignerons le besoin de coordination internationale qui en résulte et tenterons de cerner, en ordre de grandeur, les principaux enjeux qui en découlent pour la France et son industrie.

\section{De Kyoto à Buenos-Aires : dix mois pour fixer les règles du jeu}

La Conférence de Kyoto s'est structurée autour de deux lignes de confrontation géographiques :

- au sein de l'Annexe 1, des débats très vifs ont eu lieu sur le niveau des objectifs et la nature des systèmes de PEN. Ils ont opposé l'Union Européenne aux USA et au reste du JUSSCANZ (Canada, Australie, Nouvelle-Zélande), le Japon et la Russie ayant un rôle spécifique, mais moins déterminant;

- entre pays développés et pays «hors Annexe 1 », les négociations ont porté sur la mise en place de mécanismes susceptibles d'intéresser les pays en développement à participer à l'effort. Le mandat de Berlin ne prévoyait aucun engagement de leur part, mais après un vote unanime du Sénat américain avertissant qu'aucun accord ne serait ratifié dans ces conditions, une porte de sortie fut recherchée en direction de discussions ultérieures à ce sujet, en échange d'un Clean Developement Fund (qui pourrait être le FEM réformé), ou de mécanismes d'application conjointe permettant à des industries des pays de l'Annexe 1 de procéder à des abattements dans les pays hors Annexe et de les comptabiliser.

Le résultat le plus spectaculaire de la Conférence est l'adoption d'objectifs ambitieux de réduction des émissions, par rapport à 1990, à un horizon compris entre 2008 et 2012 : - 8\% pour l'UE (bulle au sein de laquelle opèrera une différenciation), $-7 \%$ pour les USA et $-6 \%$ pour le Japon, 0\% pour la Russie. La référence à 1990 est ici trompeuse puisque ces chiffres correspondent par exemple à -21\% pour les USA par rapport aux émissions 1996 et bien plus par rapport aux projections 2010. En revanche la Russie est actuellement à quelques $30 \%$ en dessous de son niveau 1990 en raison de sa crise interne, ce qui posera un problème majeur pour l'organisation des marchés internationaux de PEN, on le verra. Ces chiffres sont en fait le point moyen entre la proposition initiale de l'UE (moins $15 \%$ homogènes pour tous) et des USA (stabilisation et hétérogénéité des objectifs), rapprochement facilité par la prise en compte de six gaz au lieu de trois dans le calcul ${ }^{3}$ et le report de l'échéance à 2012 au lieu de 2010. On notera que la différenciation symbolique entre pays s'est faite mais sans référence à une règle quelconque.

Ces objectifs ont pour vocation de devenir contraignants après ratification de l'accord par deux-tiers des Parties, mais cette ratification ne pourra intervenir sans que soient préalablement précisés des éléments de règles du jeu concernant les sanctions, les PEN, et un Mécanisme de Développement Propre en direction des pays du Tiers-Monde, éléments sur lesquels aucun accord n'a pu intervenir à temps.

Une opposition subsiste en effet entre l'Europe et le Japon d'un côté, les USA et le JUSSCANZ de l'autre, quant à la nature des règles minimales susceptibles de gouverner le lancement de systèmes de PEN, et surtout quant au fait de savoir si des échanges internationaux pourraient avoir lieu entre firmes dès maintenant. Pour comprendre l'enjeu de

\footnotetext{
${ }^{3} \mathrm{CO}_{2}, \mathrm{CH}_{4}, \mathrm{~N}_{2} \mathrm{O}, \mathrm{HFCs}, \mathrm{PFCs}, \mathrm{SF}_{6}$.
} 
ce débat, il faut préciser que seules les Parties (les gouvernements) détiennent des permis mais qu'elles peuvent déléguer leurs pouvoirs, sous leur contrôle et responsabilité, à des entités privées ; stricto sensu, les échanges de permis ne peuvent donc commencer qu'au début de la première période budgétaire, soit en 2008. Mais, on ne peut évidemment concentrer tous les abattements sur cette période et il incurver bien avant les tendances; les USA veulent donc que les entreprises puissent rapidement acheter des contrats à terme sur les PEN, et les échanger, ce qui suppose que des échanges internationaux de tels «futures » soient reconnus licites. Le conflit porte alors sur le fait de savoir s'il faut préalablement s'entendre sur des règles (position européenne critiquée par les USA, qui craignent d'être entraînés dans des discussions sans fin sur des systèmes complexes qui gèleraient en fait les marchés), ou si ces échanges pourraient commencer dès aujourd'hui, au risque de mettre la future négociation des règles devant un fait accompli. Le compromis s'est fait sur une rédaction prévoyant la négociation des règles dès la prochaine Conférence des Parties (COP4) à Buenos Aires en novembre 1998.

En revanche, les pays développés n'ont pu proposer aux pays en développement une proposition crédible qui permettrait un mouvement de leur part. Les propositions de «Fonds » supposent en effet qu'il y ait un financement public dont on s'assure qu'il soit additionnel par rapport aux aides publiques existantes (préoccupation des pays du Tiers-Monde). Devant l'échec des tentatives pour trouver des formules minimisant les risques de sélection adverse dans l'usage des fonds du GEF (Global Environment Facility), et dans un contexte de limitation des dépenses publiques disponibles dans les pays donneurs, les sommes susceptibles d'être réunies sont hors de proportion avec l'enjeu et peu susceptibles d'intéresser les PVD. L'application conjointe impliquant le secteur privé apparaît alors comme susceptible de déclencher des transferts plus massifs d'investissement et de technologie (systèmes électriques, réseaux de distribution de gaz, assainissement des décharges, qualité thermique des bâtiments, etc...) vers des pays comme la Chine, l'Inde et le Brésil qui sont ici les trois pays clefs du G77. L'impasse du système est qu'aucune formule n'est aujourd'hui disponible pour pallier l'inobservabilité des émissions de référence et pour éviter des réductions fictives : dans les pays en développement en effet les infrastructures sont en expansion rapide et retenir l'écart entre technologie existante et technologies nouvelles comme critère du système peut revenir à comptabiliser des réductions qui auraient eu lieu de toute façon. En dehors de son détournement des objectifs climatiques, l'application conjointe peut donc avoir pour résultat de biaiser les marchés en incitant les entreprises à privilégier les opérations où les effets d'aubaine sont les plus abondants. Devant l'opposition très vive de la Chine et de l'Inde à la mention même des termes « application conjointe » à propos des pays hors Annexe 1, et pour éviter un blocage total, l'accord s'est fait sur un néologisme, le Mécanisme de Développement Propre, dont le contenu reste indéterminé.

Formellement, Kyoto n'a ainsi rien réglé, puisque des éléments essentiels pour la mise en œuvre effective du protocole ne seront en définitive fixés qu'ultérieurement. Un échec est en principe tout à fait envisageable à Buenos Aires, ou encore un accord sur un contenu qui interdirait sa ratification par certains pays clefs, les USA en premier lieu en raison des positions prises par le Sénat. En pratique toutefois, peu d'observateurs pensent que Kyoto sera sans suite; l'annonce officielle du Vice Président Al Gore selon laquelle, accord ou pas, les USA procéderaient à une stabilisation de leurs émissions, ne saurait en effet être vue comme un effet de tribune. L'administration actuelle a manifestement fait un choix de politique intérieure, avec bien sûr une dimension géopolitique, pour enclencher une mutation du système énergétique américain, choix devant lequel Nixon, Carter et Bush avaient 
successivement dû reculer ${ }^{4}$. Elle a déjà prévu, tout au long de cette année, de mener une campagne d'explication visant à empêcher les chambres de bloquer la ratification.

Dans un tel contexte, nul ne s'attend à ce que des formules définitives soient trouvées d'ici onze mois concernant le Mécanisme de Développement Propre; il suffira que des principes généraux soient formulés, de même que les conditions d'entrée des pays en développement dans l'Annexe 1. En revanche, un accord devra être trouvé sur les PEN à Buenos Aires pour permettre à l'administration US de mener la campagne de la ratification, et son contenu déterminera les conditions de la compétition internationale.

\section{Permis d'émission négociables : quels besoins d'harmonisation ?}

Les PEN sont parfois présentés comme l'équivalent technique de taxes pigouviennes, puisqu'ils égalisent les coûts marginaux de réduction des pollutions. Ils sont aussi présentés, dans la rhétorique des négociations, comme un outil préservant la souveraineté des gouvernements, puisque n'exigeant aucune mesure complémentaire d'harmonisation. Mais ces deux propositions ne valent que dans un monde de premier rang et ignorent trois questions fondamentales : celle de l'allocation initiale des droits et de ses effets redistributifs, celle des conditions d'efficacité des marchés (questions de crédibilité, de transparence, d'apparition de rentes) et celle des effets d'équilibre général. Nous ne traiterons pas ici des questions de séparabilité entre équité et efficacité dans le contexte d'un bien public produit de façon décentralisée (Chichilnisky, Heal, Starett 1996) puisqu'on peut considérer que l'accord de Kyoto résout pour un temps la question de la distribution initiale, et nous nous concentrerons donc sur les deux derniers points qui conditionneront l'accord de Buenos Aires et ses implications pratiques.

\section{II.1. Conditions d'efficacité des marchés internationaux de PEN}

L'expérience des systèmes de PEN existant à l'échelle nationale (Hahn et Stavins, 1991) montre que leur efficacité dépend de la fiabilité des mesures et des contrôles, de l'accessibilité des marchés et de la crédibilité des sanctions. Ces critères posent des problèmes spécifiques lors de l'extension des systèmes à l'échelle internationale (Ocde 1993) et tout particulièrement le dernier puisque nous n'avons que peu d'expérience en matière de pénalités économiques infligées à des gouvernements pour cause de non respect d'engagements ; toutefois nous n'aborderons pas cette question ici, car probablement seule l'expérience tranchera (comme dans le cas de l'OMC). Nous insisterons ici sur les points sur lesquels des règles peuvent être édictées préalablement au lancement des marchés.

\section{II.1.1. Garanties de transparence :}

Le texte du Protocole de Rio prévoit qu' « une Partie pourra acquérir de ou transmettre à une autre Partie » les Qelros initialement répartis au début de la première période budgétaire. Littéralement, ceci autorise des échanges bilatéraux entre Etats, ce qui ouvre immédiatement la voie à l'abus que constitueraient des utilisations stratégiques des échanges de permis d'émission, sources de distorsions de concurrence sur d'autres marchés. Ce danger est renforcé par la situation spécifique de la Russie et des autres pays en transition. Certes, on peut considérer qu'en 2008 ces pays auront recouvré une croissance économique durable et se seront rapprochés de leurs engagements de réductions. Mais, partant d'une situation en 1996

\footnotetext{
${ }^{4}$ Sur la liaison entre environnement global et stratégie énergétique, on se reportera ici à l'allocution de J. Schlessinger lors de la Conférence Mondiale de l'Energie à Montréal en 1989.
} 
qui correspond à $70 \%$ environ de leurs émissions en 1990, il est fort possible qu'ils se retrouvent en possession de quantités de PEN correspondant à des réductions fictives (du «hot air» dans le jargon en vigueur) et échangeables contre de grands contrats d'infrastructure, dont le marchandage est déjà partiellement politisé. La seule façon d'assurer une transparence des marchés et de s'assurer de la vérité des prix internationaux est ici que tout achat ou vente par un gouvernement (à un autre gouvernement ou à une entreprise) passe par un appel public ou mieux une bourse internationale (chambre de compensation).

Le même problème se pose dès aujourd'hui pour les échanges entre entreprises à l'échelle internationale. Il convient en effet d'assurer un libre accès aux marchés à terme, en particulier pour les entreprises de petite taille ou situés dans des pays ne pouvant mettre en place des structures d'information sur la réalité des échanges dans les grands pays acheteurs ou vendeurs. Là encore, pour éviter les abus de position dominante (avec des arrangements bilatéraux qui assèchent les marchés dans certaines régions) il conviendrait de mettre en place des bourses d'échange des droits. Mais le débat porte sur le fait de savoir si le passage en bourse doit être obligatoire, certains craignant que l'interdiction d'échanges bilatéraux n'inhibe l'éclosion des marchés.

\section{II.1.2. Responsabilité légale, traitement du « hot air » et sanctions par effet de réputation:}

Jusqu'ici, la pratique américaine quant aux systèmes de PEN déjà en place aux USA consiste à reporter sur le vendeur la responsabilité de la cession de droits correspondant à des abattements fictifs. On peut se demander ici si la charge du risque de transaction ne devrait pas peser en partie sur l'acheteur aussi pour l'inciter à la circonspection. Certains des pays vendeurs en effet, comme la Russie, sont des pays où l'Etat de droit a de la peine à s'affirmer et dans lesquels la fiabilité des systèmes de mesure risque d'être problématique, dans un premier temps au moins. Or ce sont les pays dans lesquels la question du «hot air » se pose de façon cruciale. Dès lors, devant la difficulté de mettre en place des sanctions économiques effectives par voie légale, la menace d'une sanction possible sur l'acheteur peut créer un mécanisme de sanction effective par effet de réputation, au même titre que les décotes sur créances douteuses.

\section{II.1.1. Efficacité et harmonisation des modalités d'allocation interne des droits}

On doit distinguer ici deux interrogations: quels acteurs économiques détiennent les permis d'émettre et sous quelle forme leur sont-ils alloués par les Etats?

En théorie, il existe au moins deux grandes options pour l'allocation des permis :

- allocation aux émetteurs de GES ; en raison de coûts de transaction qui deviendraient vite exorbitants cette option ne peut qu'être limitée aux grands secteurs émetteurs (couplée par exemple à une taxe pour les sources non concentrées, dont les consommations de carburant);

- allocation à ceux qui font rentrer du carbone dans l'économie, c'est-à-dire aux importateurs ou producteurs d'énergie fossile ; cette option a l'avantage de fournir un signal-prix général qui se transmettrait en aval à tous les utilisateurs. Mais elle présente la faiblesse de faire gérer la contrainte par ceux qui ne disposent pas d'une information adéquate sur les potentiels d'abattement chez les utilisateurs (on retrouve le problème bien connu de la gestion de l'efficacité énergétique sur les usages finaux). 
Concernant les modes d'allocation, il existe aussi deux variantes : l'allocation gratuite ou la vente aux enchères. La première option est la seule a avoir été utilisée jusqu'ici, en général au pro rata des émissions de l'année de base (benchmarking). Mais elle ne peut être appliquée qu'à un nombre restreint d'entreprises fonctionnant souvent dans des secteurs à demande peu élastique aux prix, d'où le risque d'apparition de rentes. Ces rentes peuvent être considérées comme une compensation de l'effort imposé aux industries énergétiques et intensives en énergie et une façon de faciliter leur adaptation au nouveau contexte. Mais elles constituent une perte d'efficacité statique et surtout une perte d'efficacité dynamique en diminuant l'incitation à investir dans des technologies à basse intensité en carbone, puisque l'innovation en ce domaine vient éroder la valeur des permis détenus (Toman 1998). La vente aux enchères permet de contourner ces difficultés, mais peut paraître difficile à mettre en œuvre. Une voie moyenne revient à distribuer gratuitement les permis puis à taxer les rentes obtenues (Haites 1997).

L'existence de ces variantes fait que l'instauration de systèmes de PEN ne résout pas ipso facto les problèmes de distorsions de concurrence et de souveraineté nationale. Un pays A qui choisirait de vendre ses PEN aux enchères ou qui opterait pour un système d'écotaxes pénaliserait ses entreprises intensives en énergie par rapport à un pays B qui les distribuerait gratuitement. En d'autres termes, chaque pays reste formellement libre de ses choix, mais ceux-ci risquent d'être fortement contraints si se développent rapidement des PEN distribués gratuitement dans certains pays clefs. En outre, si les gouvernements sont totalement libres des règles de ré-allocation interne, ils peuvent en avoir un usage stratégique favorisant tel ou tel secteur industriel selon les rapports de force prévalant dans le pays. On peut arguer qu'après tout les gouvernements ont bien d'autres moyens d'opérer ces distorsions de concurrence (financement, recherche, influences politiques), mais on ne peut dès lors garantir que les PEN internationaux conduiront à une allocation optimale des moyens. Ceci est vrai en optimum partiel (minimisation des coûts techniques d'abattement mais l'est encore plus si on considère les effets d'équilibre général.

\section{2. Evaluation des PEN du point de vue de leurs effets d'équilibre général}

Les analyses comparées des effets d'équilibre général de divers outils d'incitation publique font apparaître qu'à réductions d'émissions égales l'impact net de PEN sur une économie est plus négatif que celui d'écotaxes ou de PEN aux enchères. I. Parry, R. Williams et L. Goulder (PWG) montrent ainsi que, dans le cas américain, le coût d'une réduction de 5\% des émissions de carbone par l'instauration de quotas est sept fois supérieur au même coût en cas de recours à la taxation, celui d'une réduction de $15 \%$ encore trois fois supérieur. Ce résultat s'explique en partie par le montant de la rente dégagée par les industries énergétiques et les distorsions de concurrence au détriment des entreprises nouvelles en cas de quotas. Mais le déterminant majeur de l'écart est le recyclage des produits de la taxe ou des enchères, soit le dividende économique que ces produits peuvent entraîner. Ainsi pour D. Jorgenson (1997) : "If the revenues from an hypothetical carbon tax were rebated to consumers on the form of reduced taxes on wage and salary income, the cost in the worst case (above of 1.6 percent of the GDP) would be reduced to 0.6 percent, or by a factor three. But if taxes were rebated on capital income instead, the loss would turn into a gain ».

Ce mécanisme, désigné dans la littérature sous le terme de double-dividende, a pu être contesté sous sa forme «forte » (Bovenberg, Van des Mooj 1994, Goulder 1994) c'est-à-dire quant à sa capacité d'engendrer un gain net en croissance et emploi. Les arguments contre la forme forte du double-dividende font valoir que les écotaxes ont un coût économique net en 
raison du mécanisme suivant : elles introduisent des distorsions sur le marché des biens finaux d'où une baisse de l'utilité d'un revenu donné, et cet effet n'est pas compensé par une baisse des coûts de production, puisque les écotaxes retombent en dernière instance sur les facteurs primaires donc le travail ; les travailleurs enregistrent ainsi une baisse de leur revenu réel et arbitrent alors pour le loisir, d'où une contraction de la demande finale des biens et services marchands. Les modèles incorporant un progrès technique induit soulignent en outre un effet d'éviction entre investissements sur les technologies propres et les autres investissements, mais ce mécanisme existe aussi (et plus fort) dans le cas de PEN gratuits.

Ces arguments peuvent être discutés à plusieurs niveaux comme le caractère distorsif, pour le consommateur des taxes existantes ou les effets du nouveau partage de la rente pétrolière ou gazière entre exportateurs et importateurs, partage qui est crucial pour un pays comme le nôtre. Mais c'est sur le comportement du marché du travail que la discussion se joue en fait l'essentiel des conclusions sur le double-dividende. En représentant un monde avec flexibilité totale du travail et adaptation des salaires, où le problème de l'insuffisance de la demande finale n'est pas posé et où les salariés ne recourent pas à l'économie parallèle ou domestique pour contrebalancer des pertes de pouvoir d'achat, bien des modèles d'équilibre général manquent deux éléments centraux dans le contexte européen:

- une écotaxe sur le $\mathrm{CO} 2$, recyclée dans une baisse des charges sociales, découpler le financement de la protection sociale de la formation des coûts de production. Payée en partie par les consommateurs, elle se traduit, coeteris paribus, par une baisse des coûts de production moyens.

- les modèles existants ont une approche comptable de la substitution entre tel ou tel mode de prélèvement et ignorent les effets dynamiques des prix relatifs des facteurs de production sur les comportements d'embauche des entrepreneurs en présence d'incertitude concernant le niveau final de leurs ventes. Sauf à admettre une flexibilité totale du travail, toute décision d'embauche revient en effet pour l'entrepreneur à accepter un coût fixe supplémentaire. On peut alors montrer aisément qu'une taxe sur le travail tombe sur les sureffectifs et aggrave donc les cycles baissiers ; au contraire, une taxe sur les produits polluants est corrélée avec la conjoncture économique. La substitution entre écotaxes et prélèvement sur le travail conduit donc à réduire la taxe implicite sur les sureffectifs et la prise de risque des entrepreneurs lors de l'embauche.

Au total, l'ampleur du double-dividende associé à une écotaxe est donc surtout fonction du jugement que l'on porte sur les distorsions dynamiques de la fiscalité actuelle sur le marché du travail. En fait, contesté sous sa forme forte, le double-dividende d'écotaxes ou de PEN aux enchères n'est en revanche pas contesté sous sa forme « faible », c'est-à-dire quant à sa capacité à baisser le coût économique net des politiques environnementales. Or c'est bien cet avantage qui risque d'être annihilé en cas de PEN gratuits.

\section{Conséquences pour l'économie et l'industrie française}

La principale leçon de l'analyse économique est donc que les formes de lancement des marchés de PEN devraient rester compatibles avec des réformes fiscales et supprimer tout risque de manipulation stratégique, ce qui passe par une harmonisation des formes de rétrocession aux acteurs économiques (Godard 1997). Or, il est peu probable que l'administration américaine accepte un compromis sur ce point puisque cela serait présenté par les adversaires de l'action comme anticonstitutionnel en limitant la souveraineté de l'Etat américain. C'est argument, qui fût déjà employé dans le cas des écotaxes et de l'OMC, ne 
peut être combattu que par un front uni des autres pays, ce qui est fort improbable.

Or la pression pour l'obtention de règles pour les permis susceptibles de rentrer en vigueur immédiatement a pour limite le fait que le Protocole de Kyoto, en raison du précédent de la «bulle» européenne qui organise une différentiation interne autour d'un objectif commun, ouvre la possibilité d'ouverture d'autres «bulles ». Les USA pourraient en effet menacer de s'associer à d'autres pays, la Russie par exemple en cas de demandes trop pressantes sur l'harmonisation des règles: les émissions de cette bulle devraient égaler la somme des engagements individuels initiaux, mais les USA pourraient demander une hausse de leurs droits en échange de soutiens financiers ou technologiques. Ce troc global peut certes s'avérer politiquement difficile à opérer, mais sa menace est suffisamment forte pour envisager sérieusement l'hypothèse où la $\mathrm{COP}$ de Buenos Aires n'adoptera pas de clause sur l'harmonisation des règles de rétrocession. Le bref exercice numérique ci-après essaie d'en cerner les conséquences pour la France en examinant en quoi, dans le cas le plus défavorable, serait affectée sa marge de manœuvre en matière de réforme fiscale écologique. Il le fait en se plaçant dans l'hypothèse la moins favorable où la France adopterait un écotaxe (ou des PEN vendus aux enchères), alors que les autres pays procéderaient à une distribution gratuite des permis. De plus, on majorera les coûts que devrait supporter notre économie en considérant que les autres économies pourraient annuler les coûts induits par des PEN gratuits à recourant à des montages diplomatiques permettant d'accéder à des réductions fictives, du « hot air» dans les pays en transition, et ceci en quantité suffisante.

\section{III.1. PEN versus écotaxes : analyse des effets d'équilibre général}

Nous résumons ici un exercice présenté in (Hourcade, Ghersi 1997). Il s'agit d'un exercice de statique comparative qui vise à déterminer le nouvel équilibre de long terme qui s'établit après ajustement suite à des mesures de taxation ou l'établissement de PEN. Cet équilibre dépend principalement des élasticités-prix à long terme de la demande d'énergie pour les ménages et l'industrie, de l'effet d'éviction entre investissements d'abattement des émissions et autres investissements, de la boucle prix-salaires et des conséquences de la transformation des prix relatifs sur la compétitivité internationale.

En partant d'émissions globales de 116 millions de tonnes de carbone en 2010, ce qui correspond au scénario central du Commissariat Général du Plan, l'écotaxe nécessaire pour tenir l'engagement initial de la France au sein de la bulle européenne (et qui est susceptible d'être légèrement réajustés après Kyoto) serait de $850 \mathrm{FF}$ par tonne de $\mathrm{CO} 2$. Si on recycle son produit sous forme de baisse des charges sociales pesant sur les coûts salariaux (somme des cotisations patronales et salariales), on obtient une baisse de $5 \%$ de ces charges, une hausse de la consommation finale des ménages de 0,12 à $0,36 \%$ et entre 182000 et 206000 emplois nouveaux. Ces chiffres sont bien sûr discutables (ils ne prennent pas en compte les effets de chocs sectoriels et, dans l'autre sens, la baisse du risque à l'embauche) et ne visent qu'à examiner comment le bilan économique d'ensemble se déplace entre une écotaxe et des PEN gratuits pour un même jeu d'hypothèses.

Dans le cas où la France adopterait des PEN gratuits, on retrouve ici les résultats déjà cités de Goulder et Jorgenson : si par hypothèse le prix international des permis s'établit à $300 \mathrm{~F}$ par tonne de carbone, la baisse de consommation des ménages est de $-0,05$ à $-0,10 \%$ et la perte d'emplois de 100000 environ ce qui s'explique très logiquement par l'absence du double-dividende engendré par le recyclage des produits de l'écotaxe. Logiquement l'Etat pourrait donc lever une taxe intérieure forte, même en cas de PEN gratuits à l'extérieur tant 
que le mécanisme vertueux qui préside au double-dividende fonctionne (celui-ci s'annule pour des écotaxes supérieures à $2200 \mathrm{FF} / \mathrm{t}$ dans les conditions du modèle). En fait, cette marge de manœuvre est limitée par le fait que des distorsions trop importantes sur la compétitivité des industries lourdes peuvent induire des chocs économiques difficiles à gérer à l'échelle régionale. Il y a donc bien ici un risque de limitation des marges de manœuvre en matière de fiscalité et de double-dividende qu'on ne peut apprécier qu'en passant à une analyse sectorielle.

\section{III.2. Risques de distorsions de concurrence}

Trop souvent l'étude des impacts d'une taxe-carbone se fait sans étude symétrique les distorsions de concurrence liées aux taxes sur l'énergie et celles liées aux taxes sur le travail et sans prise en compte des adaptations technologiques (Giraud, Nadaï 1997). Pour fixer les ordres de grandeurs nous avons, au prix d'approximations acceptables dans un tel contexte, calé un raisonnement sectoriel sur les résultats agrégés présenté ci-dessus en examinant comment se transforme le bilan net par secteur au fur et à mesure de l'introduction de tous les effets d'équilibre général induits par le changement technique et le recyclage du produit des écotaxes.

Dans le «bilan I », on présente les surcoûts par branche industrielle d'une taxe de 850 $\mathrm{FF} / \mathrm{tC}$ portant sur toutes les émissions, mais sans recyclage des produits de la taxe. On obtient des surcoûts de plus de 3\% pour les industries agricoles et alimentaires, la production de coke, l'industrie pétrolière, la première transformation des métaux ferreux, la chimie de base et les transports. Le «bilan II », simule une taxe prélevée seulement sur le secteur industriel mais recyclée par une baisse des charges sociales et en tenant compte de l'adaptation technologique: les branches les plus touchées voient leurs surcoûts baisser notablement (une réduction d'un point en moyenne) mais restent fortement perdantes. L'ensemble des firmes perdantes représentent $14 \%$ de la production distribuée et $10 \%$ de la masse salariale. Dans le «bilan III », on simule une taxe sur l'ensemble des émissions, y compris les ménages, mais on raisonne sans changement technique. Les charges pesant sur le entreprises baissent de 1,8 GF ce qui explique que seuls quatre secteurs subissent des surcoûts compris entre $1 \%$ et $3 \%$ : production de pétrole et gaz naturel, chimie de base, minerais ferreux et première transformation de l'acier, cokéfaction. En revanche les perdants représentent $18 \%$ de la production distribuée et $13 \%$ de la masse salariale.

Le «bilan IV », qui regroupe tous les effets précédents en tenant compte d'une taxation de toutes les émissions, d'un recyclage intégral dans une baisse des charges sociales et du changement technique ; il donne la vraie mesure des enjeux. En dehors de la branche pétrole et dérivés les surcoûts restent inférieurs à $1 \%$ et les «perdants » ne représentent plus que $9 \%$ de la production distribuée et $4 \%$ de la masse salariale.

\section{Conclusion}

L'exercice précédent permet de cerner les enjeux de politique publique après Kyoto en tenant compte des incertitudes qui pèsent sur les modalités d'applications du Protocole de Kyoto. En effet, le lancement à grande échelle de PEN gratuits est loin d'être acquis même en l'absence de contraintes sur ce point à Buenos Aires. D'une part bien des économistes américains insistent sur les risques de tels systèmes et privilégient les permis aux enchères voire le écotaxes; d'autre part, si des règles de transparence et bon fonctionnement des marchés sont adoptées, il n'est pas évident que le déploiement de marchés internationaux de 
PEN soit très rapide ne serait - ce qu'en raison de l'ampleur supposée mais controversés de gisement de réduction à coûts négatifs aux USA. Mais même dans l'hypothèse inverse, on peut imaginer une combinaison entre une écotaxes pour les émissions dispersées (industries faiblement émettrices, transport, domestique et tertiaire) et de permis négociables internationaux pour la frange des gros émetteurs que ne représentent en fait que $16 \%$ environ des émissions et $4 \%$ de la masse salariale. La limitation des marges de manœuvre en matière de réforme fiscale et de double-dividende serait donc faible, et ce d'autant plus que seraient retirées de l'assiette des écotaxes des activités qui, de toute façon n'aurait jamais pu y être incluses en raison de leur poids politique et économique.

En fait, les simulations précédentes sont trop frustes pour qu'on puisse en tirer plus que des éléments de cadrage. Elles ont le mérite cependant de souligner à quel point les effets de distorsion de la compétitivité par les coûts en cas de non harmonisation des règles sur les échanges internationaux de PEN sont finalement de second ordre. Les effets de distorsions les plus importants risquent en effet de venir des asymétries dans les accès aux marchés d'abattement et dans les modifications induites dans les flux de capitaux, ce qui est d'ailleurs confirmé par les premières simulations du modèle G-cube de Wilcoxen. La négociation sur les règles de transparence des marchés et sur les questions de responsabilité légales seront donc en fait plus importantes que celles sur l'harmonisation des modes de rétrocession interne.

Nous voudrions terminer ce texte par quelques brèves remarques concernant l'agenda pour la recherche. Jusqu'ici en effet le type de sujet dont nous venons de traiter était l'apanage de l'économie de l'environnement conçue comme une branche de l'économie publique. Ni l'économie industrielle, ni la macro-économie ne les prenaient vraiment en charge. Or, nous venons de voir que, pour les traiter, il est nécessaire de recourir à des analyses en équilibre général, mais que ces analyses sont en fait limitées par le peu de capacité que nous avons de les informer par une connaissance précise des comportements en matière d'embauche, d'adoption des technologies ou de réponse dynamique à des problèmes de compétitivité internationale. Ceci ne signifie pas que de telles connaissances n'existent pas; elles sont probablement disponibles mais il y a eu, pour cause de spécialisation professionnelle, un manque d'effort pour les intégrer de façon à donner une portée plus opératoire aux analyses existantes sur le fonctionnement des marchés de droits et sur les effets d'équilibre général. Il est frappant par exemple de constater le manque d'analyses sur des marchés de PEN qui intègrent la réalité des relations amont - aval dans les filières industrielles ou qui puissent resituer le fonctionnement réel des PEN dans le cadre des dynamiques industrielles en cours (mutations technologiques et tendances en matière de localisation).

Ceci signifie que les économistes de l'environnement ne peuvent plus travailler indépendamment des macro - économistes, des économistes du travail et des spécialistes de l'économie industrielle. Cela signifie aussi que les questions d'environnement ne devraient plus être renvoyées à une province marginale de la discipline et soient perçues pour ce qu'elles sont, un champs problématique qui renvoie à des enjeux centraux pour les dynamiques industrielles, les questions d'emploi et de croissance dans les prochaines années.

Ces travaux ont pu être menés grâce au soutien que le Ministère de l'Environnement, l'Ademe et le Pir-Environnement et le programme Ecotech du Cnrs accorde au GDR-Ö̈kia 

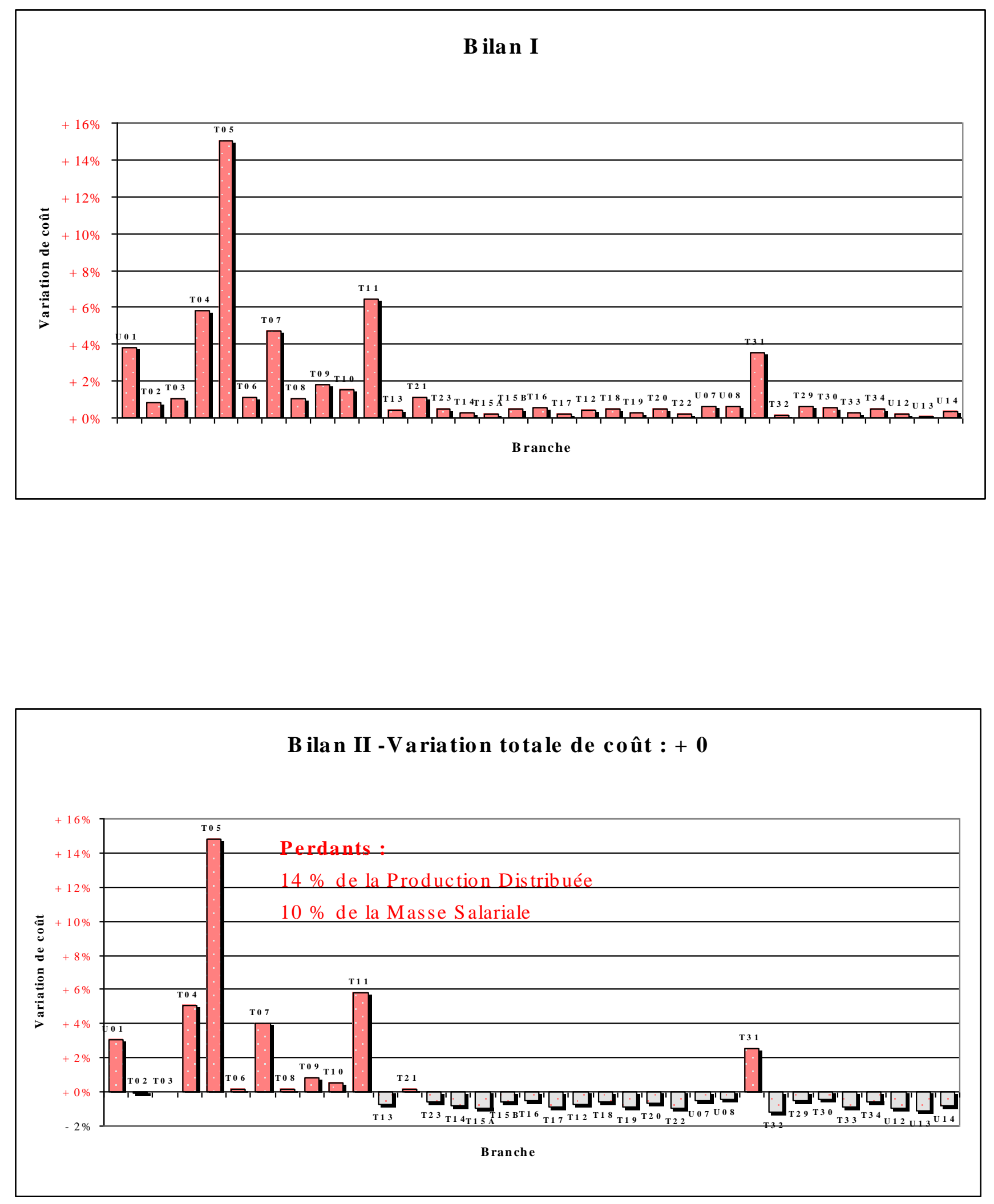

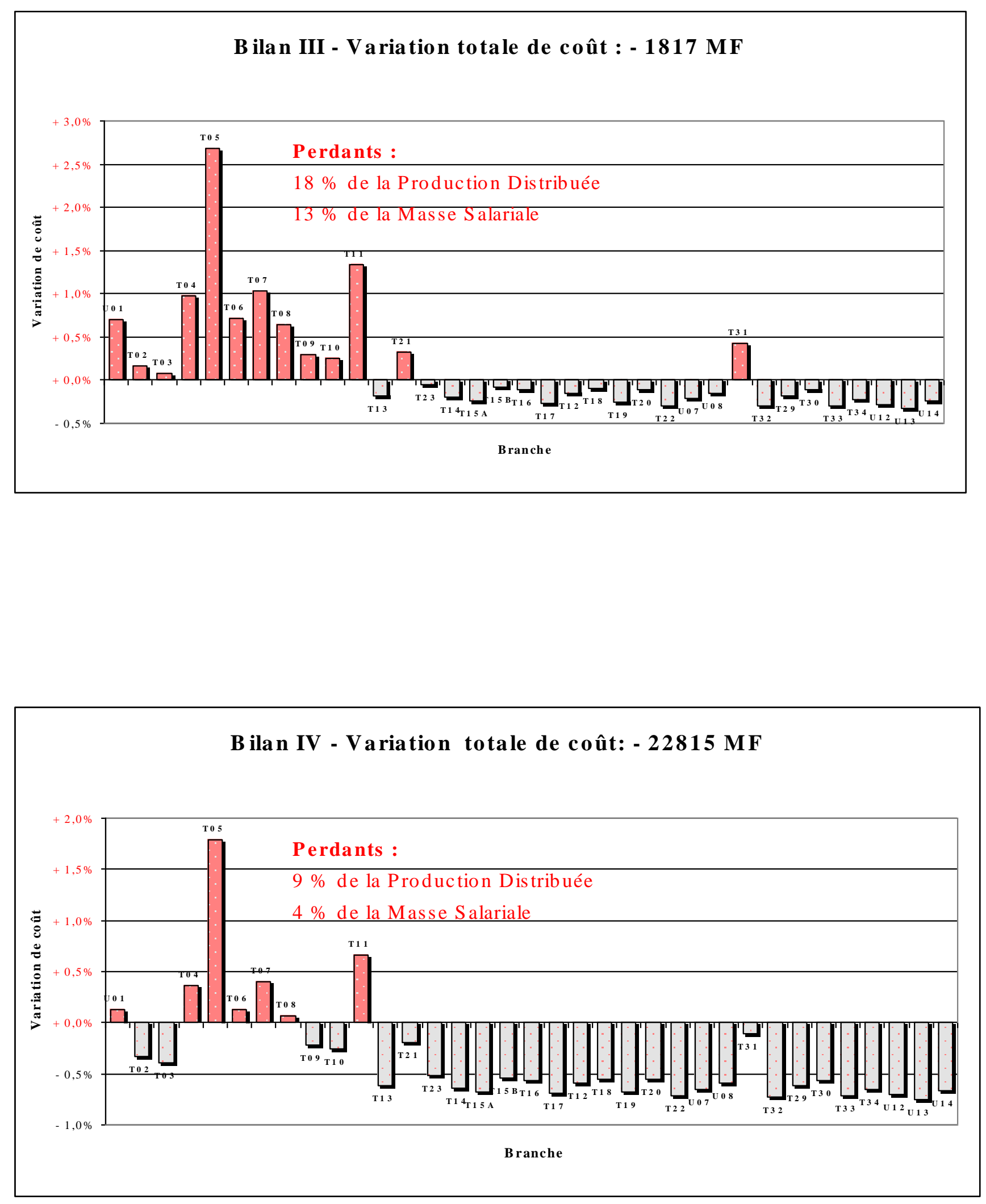


\section{Références bibliographiques}

Bovenberg A.L. et Van des Mooj R.A. (1994), Environmental Levies and Distortionary Taxation, in American Economic Review n ${ }^{\circ} 84$, pp. 1085-1089.

Giraud P-N, Nadaï A. Les effets économiques de la taxe carbone énergie sur l'industrie française, Futuribles, juillet-août 1994

Godard O. (1997), Les Permis Négociables et la Convention sur le Climat : de l'Expérience américaine aux Enjeux de l'Harmonisation, in Revue de l'Energie n491, pp. 606-622.

Goulder L.H. (1994), Environmental Taxation and the Double Dividend: A Reader's Guide, in 50th Congress of the International Institute of Public Finance, Session 1, Harvard University.

Hahn R.H. et Stavins R. (1991), Incentive-Based Environmental Regulations : A New Era from an Old Idea?, in Ecology Law Quarterly, n ${ }^{\circ}$, pp. 1-42.

Haites E. (1997), Implications for Canada of International Emissions Trading for Greenhouse Gases, draft of part II.

Hourcade J.C. (1997), Ecotaxes ou permis d'émission négociables: jeux de miroirs déformants, in Les cahiers de Global Chance, $\mathrm{n}^{\circ} 9$

Hourcade J.C., Ghersi F., (1997) Les enjeux des QELROS : entre logique économique et logique de négociations, in Revue de l'Energie, $\mathrm{n}^{\circ} 491$

Dale Jorgenson, How Economics Can Inform the Climate Change Debate in American Enterprise Institute for Public Policy Research, Conference Summary, May 1997.

OCDE (1993), Chap. I. Permis d'Emission Négociables, in Les Instruments Economiques Internationaux et le Changement Climatique, Paris, Ed. de l'OCDE, pp. 11-44.

Toman M. (1998), Frontiers of the Research on the Economics of Climate Change, à paraittre in European Environmental Resource Economics Journal. 